\title{
A GENERAL SYSTEM OF LINEAR EQUATIONS*
}

BY

A. J. PELL

By virtue of the Hellinger-Radon integral, $\uparrow$ which reduces in one special case to the Hellinger integral and in another special case to the sum of squares, a system of linear equations of the form

$$
x_{i}=\sum_{k} a_{i k} x_{k}+\sum_{\beta} \int_{a}^{b} \frac{d a_{i}^{(\beta)}(t) d u^{(\beta)}(t)}{d a_{0}^{(\beta)}(t)},
$$

$$
\Delta u^{(a)}(s)=\sum_{k} \Delta \bar{a}^{(\alpha)}(s) x_{k}+\sum_{\beta} \int_{a}^{b} \frac{d_{t} \Delta A^{(\alpha, \beta)}(s, t) d u^{(\beta)}(t)}{d a_{0}^{(\beta)}(t)},
$$

which involves a finite or denumerably infinite number of unknowns $\left\{x_{2}\right\}$ and $\left\{u^{(a)}(s)\right\}$, is contained in the single equation

$$
\mathfrak{u}=A[\mathfrak{u}],
$$

where $\mathfrak{U}$ is a function on a composite range and $A$ is a linear functional transformation We consider in this paper the solution of such equations, the corresponding non-homogeneous and adjoint equations, both when the functional transformation $A$ is completely continuous but not necessarily symmetric, and when it is limited and symmetric. The method used by us is the construction of an orthogonal system of functions on a composite range, and by means of this orthogonal system the transformation of the equations above into the linear equations

$$
z_{i}=\sum_{k} b_{i k} z_{k}
$$

in denumerably infinitely many unknowns in a Hilbert Space. Then the results of Hilbert's fourth memoir are simply carried over into the more general situation.

In his memoirsł and lectures E. H. Moore has considered general systems of linear equations in General Analysis, which include for example the mixed linear integral equation. While a special case of $(a)$ is the mixed linear

* Presented to the Society, December, 1917.

$\dagger$ Radon, J., Absolut additive Mengenfunktionen, $\mathrm{W}$ i e $\mathrm{n}$ er $\mathrm{A} \mathrm{k}$ a de mie Sit z u ngsb e r i c h t e, vol. 122, Abt. 2a, Heft 6-10 (1913), pp. 1295-1438.

$\ddagger \mathrm{E}$. H. Moore, On the foundations of the theory of linear integral equations, B ull e t i n of the American Mathematical Society, vol. 18 (1912), pp. 334-362. 
integral equation, that theory is not contained in this paper, for the functional transformation $A$ is not limited in the sense defined here.

Following the lines of Hilbert's theory, J. Radon* develops a theory for linear equations of the form $(b)$ where $\mathfrak{U}$ is a function on a simple range. His theory is confined to the completely continuous case, and yields at the same time the Hilbert theory of linear equations in denumerably infinitely many unknowns, and the theory of Fredholm's linear integral equations.

Section 6 deals with the properties of orthogonality and closure of a general matrix $L=\left(l^{(a, \beta)}\left(E, E^{\prime}\right)\right)$, which may consist of $\left(l_{i k}, p_{i}^{(\alpha)}(s)\right.$, $\left.\pi_{k}^{(\beta)}(t), \Lambda^{(a, \beta)}(s, t)\right)$ and includes as special cases the matrices formed from the coefficients of orthogonal systems of linear forms and linear differential forms. In his lectures E. H. Moore has given similar results for matrices in General Analysis.

I wish to acknowledge some helpful suggestions in regard to the HellingerRadon integral from Mr. T. H. Hildebrandt.

\section{Definitions}

In a space of $n$ dimensions let $I$ be defined by $a_{i} \leqq c_{i} \leqq x_{i}<d_{i}<b_{i}$ and let $\&$ be a class of sets $E$ of points of $I$ such that

(1) If $E_{1}$ and $E_{2}$ belong to (E, the sum $E_{1}+E_{2}$ and the product $E_{1} E_{2}$ belong to $\mathbb{E}$.

(2) If $E_{1}, \cdots, E_{n}, \cdots$ belong to $\&$ and are mutually distinct, $\sum_{n} E_{n}$ belongs to $E$.

We consider real single-valued functions $f(E)$ on $\mathbb{E}$.

A function $f(E)$ is absolutely additive if, when $E_{1}, \cdots, E_{n}, \cdots$ belong to $E$ and are mutually distinct

$$
f\left(\sum_{n} E_{n}\right)=\sum_{n} f\left(E_{n}\right) .
$$

A function $f(E)$ is monotonic if $f(E) \geqq 0$.

A monotonic, absolutely additive function $f_{0}(E)$ is called a basis function of the absolutely additive function $f(E)$, if $f(E)=0$ when $f_{0}(E)=0$.

The range of the variables $i, k, \alpha$, and $\beta$ is that of a sequence, which may be finite in some cases.

Hellinger-Radon integral. $\dagger$ Let $f_{0}(E)$ be a basis function of the absolutely additive function $f(E)$. Divide $E$ into a finite number of distinct sets $E_{1}$, $E_{2}, \cdots, E_{n}$, and form the sum

$$
\sum_{i=1}^{n} \frac{\left(f\left(E_{i}\right)\right)^{2}}{f_{0}\left(E_{i}\right)},
$$

* Radon, l. c.

† Radon, l. c. 
the term being defined to be zero when $f_{0}\left(E_{i}\right)=0$; if this sum has a limit as $n$ is increased and the diameter of $E_{i}$ approaches zero, the limit is denoted by the Hellinger-Radon integral

and $f(E)$ is integrable $H\left(f_{0}\right)$.

$$
\int_{E} \frac{(d f)^{2}}{d f_{0}}
$$

If $f(E)$ and $f_{1}(E)$ are absolutely additive, and integrable $H\left(f_{0}\right)$ on $E$, the sum

$$
\sum_{i=1}^{n} \frac{f\left(E_{i}\right) f_{1}\left(E_{i}\right)}{f_{0}\left(E_{i}\right)}
$$

has a limit as $n$ is increased and the diameter of $E_{\imath}$ approaches zero, and the limit is denoted by

$$
\int_{E} \frac{d f d f_{1}}{d f_{0}}
$$

A system of absolutely additive functions $\left\{f_{i}(E)\right\}$, each integrable $H\left(f_{0}\right)$ on $E$, is closed, if there exists no absolutely additive function $f(E) \neq 0$, integrable $H\left(f_{0}\right)$, such that

$$
\int_{E} \frac{d f}{d} \frac{d f_{i}}{d f_{0}}=0 \quad(i=1,2, \cdots) .
$$

We denote by $\mathfrak{F}$ a system of absolutely additive functions $\left\{f^{(a)}(E)\right\}$ on $\mathbb{F}_{a}$ classes of sets of points $I_{\alpha}$ defined by $a_{i}^{(a)} \leqq c_{i .}^{(a)} \leqq x_{i}<d_{i}^{(a)}<b_{i}^{(a)}$ in spaces of finite number of dimensions. If the Hellinger-Radon integrals of these functions with basis functions $f_{0}^{(a)}(E)$ exist, and the sum

converges, we denote it by

$$
\sum_{a} \int_{I a} \frac{\left(d f^{(a)}\right)^{2}}{d f_{0}^{(a)}}
$$

$$
\int \frac{(d \mathfrak{F})^{2}}{d F_{0}}
$$

and say that $\mathfrak{F}$ is integrable $H\left(\mathfrak{F}_{0}\right)$. It can easily be shown that if $\mathfrak{F}_{\text {and }} \mathfrak{F}_{1}$ are integrable $H\left(\mathfrak{F}_{0}\right)$ the sum

$$
\sum_{a} \int_{I_{\alpha}} \frac{d f^{(a)} d f^{(a)}}{d f_{0}^{(a)}}
$$

converges, and we denote it by

$$
\int \frac{d \mathfrak{F} d \mathfrak{F}_{1}}{d \mathfrak{F}_{0}}
$$

\section{ORthogonal SYSTEMS WITH GIVEN BASIS FUNCTIONS}

Given a monotonic absolutely additive function $f_{0}(E)$ on $I$, there exists a closed system of absolutely additive, linearly independent functions 
$f_{i}(E)$, which have $f_{0}(E)$ as a basis function and are integrable $H\left(f_{0}\right)$. Divide $I$ into two mutually distinct subsets $E_{1}^{(1)}$ and $E_{2}^{(1)}$; divide each one of these sets into two mutually distinct subsets $E_{i}^{(2)}(i=1,2,3,4)$; continue this process in such a way that the diameter of $E_{i}^{(n)}$ approaches zero. Define a system of absolutely additive functions as follows

$$
f_{i}^{(n)}(E)=\left\{\begin{array}{cl}
f_{0}(E) & E=E_{i}^{(n)} \text { or any subset of } E_{i}^{(n)}, \\
0 & E \text { distinct from } E_{i}^{(n)} .
\end{array}\right.
$$

From the system $f_{1}^{(1)}, f_{2}^{(1)}, f_{1}^{(2)}, f_{2}^{(2)}, \cdots$, drop out all the functions which are zero or linearly dependent on a finite number of the preceding, and let $\left\{f_{i}(E)\right\}$ be the system thus obtained. Obviously the functions $f_{i}(E)$ are integrable $H\left(f_{0}\right)$. For any absolutely additive function $f(E)$ such that

$$
\int_{I} \frac{d f}{d f_{i}}=0 \quad(i=1,2, \cdots),
$$

it follows that $f\left(E_{i}^{(n)}\right)=0$, and hence

$$
\int_{I} \frac{(d f)^{2}}{d f_{0}}=0
$$

and $f(E) \equiv 0$, so that the system $\left\{f_{i}(E)\right\}$ is closed.

A system of absolutely additive functions $\left\{p_{i}(E)\right\}$, which are all integrable $H\left(p_{0}\right)$, forms a normalized orthogonal system with the basis function $p_{0}(E)$ if

$$
\int_{I} \frac{d p_{i} d p_{k}}{d p_{0}}=\left\{\begin{array}{ll}
1 & i=k \\
0 & i \neq k
\end{array} .\right.
$$

For every monotonic, absolutely additive function $p_{0}(E)$, a closed normalized orthogonal system $\left\{p_{i}(E)\right\}$, with $p_{0}(E)$ as a basis function can be constructed. Because, if $\left\{f_{i}(E)\right\}$ is a closed system of linearly independent absolutely additive functions with $p_{0}(E)$ as a basis function, constants $c_{i k}$ can be determined by the usual procedure, so that $p_{i}(E)=\sum_{k=1}^{k=i} c_{i k} f_{k}(E)$ satisfy the conditions (1).

By the usual method we obtain, for any normalized orthogonal system $\left\{p_{i}(E)\right\}$, Bessel's inequality

$$
\sum_{i}\left(\int_{I} \frac{d p_{i} d f}{d p_{0}}\right)^{2} \leqq \int_{I} \frac{(d f)^{2}}{d p_{0}}
$$

for any function $f(E)$ integrable $H\left(p_{0}\right)$. A special case of (2) is

and hence the series

$$
\sum_{i}\left(p_{i}(E)\right)^{2} \leqq p_{0}(E)
$$

is uniformly convergent.

$$
\sum_{i} p_{i}(E) \int_{I} \frac{\dot{a} p_{i} d f}{d p_{0}}
$$


If the orthogonal system $\left\{p_{i}(E)\right\}$ is closed

also

$$
(E)=\sum_{i} p(E) \int_{I} \frac{d p_{i} d f}{d p_{0}},
$$

$$
\int_{I} \frac{d f d g}{d p_{0}}=\sum_{i} \int_{I} \frac{d p_{i} d f}{d p_{0}} \int_{I} \frac{d p_{i} d g}{d p_{0}},
$$

for every $f(E)$ and $g(E)$ integrable $H\left(p_{0}\right)$. From this last equality it follows that

$$
p_{0}\left(E_{1} E_{2}\right)=\sum_{i} p_{i}\left(E_{1}\right) p_{i}\left(E_{2}\right) .
$$

Let $\left\{p_{0}^{(a)}(E)\right\}$ be a system, finite or denumerably infinite, of monotonic, absolutely additive functions, and $\left\{\bar{p}_{i}^{(a)}(E)\right\}$ closed normalized orthogonal systems with $p_{0}^{(\alpha)}(E)$ as basis functions. Let $L=\left(l_{i k}\right)$ be an orthogonal matrix, that is,

and

$$
\sum_{j} l_{i j} l_{k j}=\left\{\begin{array}{ll}
1 & i=k \\
0 & i \neq k
\end{array},\right.
$$

$$
\sum_{j} l_{j i} l_{j k}=\left\{\begin{array}{ll}
1 & i=k \\
0 & i \neq k
\end{array} .\right.
$$

Let $\left(l_{i k}^{(a)}\right)$ be matrices made up from the columns of $L$, so that each column of $L$ occurs in one and only one of the $\left(l_{i k}^{(a)}\right)$. The functions defined by

$$
p_{i}^{(a)}(E)=\sum_{k} l_{i k}^{(a)} \bar{p}_{k}^{(a)}(E)
$$

are absolutely additive, form a system $\left\{\mathfrak{P}_{i}\right\}$ integrable $H\left(\mathfrak{B}_{0}\right)$, and satisfy the following orthogonal relations

or

$$
\begin{gathered}
\int \frac{d \mathfrak{B}_{i} d \mathfrak{B}_{k}}{d \mathfrak{B}_{0}}= \begin{cases}1 & i=k \\
0 & i \neq k\end{cases} \\
\sum_{i} p_{i}^{(a)}\left(E_{1}\right) p_{i}^{(\beta)}\left(E_{2}\right)=\left\{\begin{array}{cc}
p_{0}^{(\alpha)}\left(E_{1} E_{2}\right) & \alpha=\beta \\
0 & \alpha \neq \beta
\end{array},\right.
\end{gathered}
$$

$$
\sum_{i}\left(\int \frac{d \mathfrak{F} d \mathfrak{B}_{i}}{d \mathfrak{B}_{0}}\right)^{2}=\int \frac{(d \mathfrak{F})^{2}}{d \mathfrak{B}_{0}} .
$$

For every sequence $\left\{x_{i}\right\}$ of finite norm, $\sum_{i} x_{i} \mathfrak{B}_{i}$ represents a function integrable $H\left(\mathfrak{B}_{0}\right)$, and

$$
\int \frac{d \mathfrak{F} d \Sigma_{i} x_{i} \mathfrak{B}_{i}}{d \mathfrak{B}_{0}}=\sum_{i} x_{i} \int \frac{d \mathfrak{F} d \mathfrak{P}_{i}}{d \mathfrak{P}_{0}} .
$$




\section{LiNEAR FUNCTIONAL TRANSFORMATIONS AND MATRICES}

In connection with a given matrix $A$ of absolutely additive functions $\left(a^{(a, \beta)}\left(E, E^{\prime}\right)\right)$, we introduce the following notations. $\mathfrak{A}_{E}^{a}$ denotes the system of functions $a^{(a, \beta)}\left(E, E^{\prime}\right)$ for given $\alpha$ and $E ; \mathfrak{A}_{E}^{\prime a}$ denotes the system of functions $a^{(\beta, \alpha)}\left(E^{\prime}, E\right)$ for given $\alpha$ and $E$. If $\mathfrak{A}_{E}^{a}, \mathfrak{A}_{E}^{\prime a}, \mathfrak{F}$, and $\mathbb{S}$ are integrable $H\left(\mathfrak{A}_{0}\right)$, where $\mathfrak{A}_{0}$ is a system of monotonic, absolutely additive functions, we define the following functional transformations and bilinear form

$$
\begin{gathered}
A[\mathfrak{F}]=A_{E}^{a}[\mathfrak{F}]=\int \frac{d \mathfrak{A}_{E}^{a} d \mathfrak{F}}{d \mathfrak{A}_{0}}, \\
A^{\prime}[\mathfrak{F}]=A_{E}^{\prime a}[\mathfrak{F}]=\int \frac{d \mathfrak{A}_{E}^{\prime a} d \mathfrak{F}}{d \mathfrak{A}_{0}},
\end{gathered}
$$

and if $A$ [S] is integrable $H\left(\mathfrak{A}_{0}\right)$,

$$
A(\mathfrak{F}, \mathbb{S})=\int \frac{d \mathfrak{F} d A[\mathbb{B}]}{d \mathfrak{A}_{0}} .
$$

If $A=\left(a^{(\alpha, \beta)}\left(E, E^{\prime}\right)\right)$ and $C=\left(c^{(\alpha, \beta)}\left(E, E^{\prime}\right)\right)$ are two matrices such that $\mathfrak{H}_{E}^{a}$ and $\mathfrak{F}_{E}^{\prime a}$ are integrable $H\left(\mathfrak{A}_{0}\right)$, the product $D=A C$ denotes the matrix formed from the elements

$$
d^{(a, \beta)}\left(E, E^{\prime}\right)=\int \frac{d \mathfrak{A}_{E}^{a} d \mathfrak{G}_{E}^{\prime \beta}}{d \mathfrak{A}_{0}} .
$$

The matrix $\Delta\left(\mathfrak{P}_{0}\right)$ is defined by the elements

$$
\delta^{(a, \beta)}\left(E, E^{\prime}\right)=\left\{\begin{array}{cc}
p_{0}^{(a)}\left(E, E^{\prime}\right) & \alpha=\beta \\
0 & \alpha \neq \beta
\end{array} .\right.
$$

Given a system $\mathfrak{A}_{0}$ of monotonic, absolutely additive functions $a_{0}^{(a}(E)$, a linear functional transformation $T[\mathfrak{F}]$ is limited with respect to $\mathfrak{A}_{0}$, if, (1) it transforms every absolutely additive function $\mathfrak{F}$ integrable $H\left(\mathfrak{A}_{0}\right)$ into an absolutely additive function $T[\mathfrak{F}]$ integrable $H\left(\mathfrak{A}_{0}\right):(2)$ there exists a constant $M$ independent of $\mathfrak{F}$ such that

$$
\int \frac{(d T[\mathfrak{F}])^{2}}{d \mathfrak{A}_{0}} \leqq M \int \frac{[d \mathfrak{F}]^{2}}{d \mathfrak{A}_{0}}
$$

It follows immediately from the second condition that for any $\mathfrak{F}_{n}$ and $\mathfrak{F}$ integrable $H\left(\mathfrak{A}_{0}\right)$ and such that

and

$$
\begin{array}{r}
\lim _{n \rightarrow \infty} \int \frac{\left(d\left(\mathfrak{F}_{n}-\mathfrak{F}\right)\right)^{2}}{d \mathscr{A}_{0}}=0, \\
\lim _{n \rightarrow \infty} \int \frac{\left(d T\left[\mathfrak{F}_{n}-\mathfrak{F}\right]\right)^{2}}{d \mathfrak{A}_{0}}=0
\end{array}
$$

$$
\lim _{n \rightarrow \infty} T\left[\mathfrak{F}_{n}\right]=T[\mathfrak{F}] \text {. }
$$


Let $\left\{\mathfrak{B}_{i}\right\}$ be a closed orthogonal system with basis function $\mathfrak{A}_{0}$, and let $\left\{c_{i}\right\}$ be any sequence of finite norm. If $T[\mathfrak{F}]$ is a limited linear functional transformation, and $\mathfrak{F}=\sum_{i} c_{i} \mathfrak{P}_{i}$ then

$$
T[\mathfrak{F}]=\sum_{i} c_{i} T\left[\mathfrak{P}_{i}\right],
$$

and hence $T\left[\mathfrak{P}_{i}\right]$ is of finite norm. In the usual wayt it can be shown that if $\mathfrak{B}$ is integrable $H\left(\mathfrak{A}_{0}\right)$

$$
\int \frac{d(\mathfrak{S} d T[\mathfrak{F}]}{d \mathfrak{A}_{0}}=\sum_{i} c_{i} \int \frac{d \mathfrak{S}^{\circ} d T\left[\mathfrak{P}_{i}\right]}{d \mathfrak{A}_{0}},
$$

and hence

$$
\int \frac{d \mathfrak{S} d T\left[\mathfrak{B}_{i}\right]}{d \mathfrak{A}_{0}}
$$

is of finite norm. An adjoint transformation $T^{*}[\mathfrak{F}]$ can be defined by

$$
T^{*}[\mathfrak{F}]=\sum_{i} \mathfrak{P}_{i} \int \frac{d \mathfrak{F} d T\left[\mathfrak{P}_{i}\right]}{d \mathfrak{A}_{0}}
$$

A matrix $A$ of absolutely additive functions $a^{(a, \beta)}\left(E, E^{\prime}\right)$, integrable $H\left(\mathfrak{A}_{0}\right)$, is limited with respect to $\mathfrak{A}_{0}$, if the functional transformation $A[\mathfrak{F}$ ], defined in (6), is limited with respect to $\mathfrak{A}_{0}$.

TheoRem I. A functional transformation $T$ which is linear and limited with respect to $\mathfrak{A}_{0}$, gives rise to a matrix $A$ of absolutely additive functions $a^{(a, \beta)}\left(E, E^{\prime}\right)$ integrable $H\left(\mathfrak{A}_{0}\right)$ for every $\alpha$ and $E$, such that

and

$$
\begin{aligned}
& T=A[\mathfrak{F}]=\int \frac{d \mathfrak{A}_{E}^{a} d \mathfrak{F}}{d \mathfrak{A}_{0}}, \\
& A(\mathfrak{F}, \mathfrak{H})=A^{\prime}(\mathfrak{H}, \mathfrak{F}),
\end{aligned}
$$

for $\mathfrak{F}$ and $\$$ such that

$$
\mid A(\mathfrak{F},(\mathfrak{H}) \mid \leqq \mathrm{M}
$$

and

$$
\int \frac{(d \mathfrak{F})^{2}}{d \mathfrak{A}_{0}} \leqq 1
$$

$$
\int \frac{(d \mathfrak{S})^{2}}{d \mathfrak{U}_{0}} \leqq 1
$$

Let $\left\{\mathfrak{P}_{i}\right\}$ be a closed orthogonal system with basis function $\mathfrak{A}_{0}$, then the matrix $B$ defined by the elements

$$
b_{i k}=\int \frac{d \mathfrak{P}_{i} d T\left[\mathfrak{P}_{k}\right]}{d \mathfrak{A}_{0}}
$$

$\dagger$ Hellinger, E., Neue Begründung der Theorie quadratischer Formen von unendlichvielen Veränderlichen, Journal fürdie reine und angewandte Mathematik, 1907, p. 239. 
is limited.* If $\left\{x_{i}\right\}$ is a sequence of finite norm, then, from (5), the linearity of $T$, property (2) of a limited functional transformation, and from ( $\left.5^{\prime}\right)$

$$
\sum_{k} b_{i k} x_{k}=\sum_{k} x_{k} \int \frac{d \mathfrak{P}_{i} d T\left[\mathfrak{P}_{k}\right]}{d \mathfrak{A}_{0}}=\int \frac{d \mathfrak{P}_{i} d \sum_{k} x_{k} T\left[\mathfrak{P}_{k}\right]}{d \mathfrak{A}_{0}}=\int \frac{d \mathfrak{P}_{i} d T\left[\sum_{k} x_{k} \mathfrak{P}_{k}\right]}{d \mathfrak{A}_{0}} .
$$

But $\sum_{k} x_{k} \mathfrak{P}_{k}$ is integrable $H\left(\mathfrak{A}_{0}\right)$, and from the third relation of (4) it follows that $\sum_{k} b_{i k} x_{k}$ is of finite norm, and $B$ is limited. The functions

$$
a^{(a, \beta)}\left(E, E^{\prime}\right)=\sum_{i, k} p_{i}^{(a)}(E) b_{i k} p_{k}^{(\beta)}\left(E^{\prime}\right)
$$

are absolutely additive in $E$ and $E^{\prime}$, integrable $H\left(\mathfrak{A}_{0}\right)$ for every $\alpha$ and $E$, also for every $\beta$ and $E^{\prime}$, and are such that

$$
T[\mathfrak{F}]=A[\mathfrak{F}]
$$

From the properties of the limited matrix $B$, the remainder of the theorem follows.

Let $\mathfrak{F}^{(n)}, \mathfrak{F}, \mathfrak{F}^{(n)}$, $\&$ be integrable $H\left(\mathfrak{A}_{0}\right), \int\left(\left(d \mathfrak{F}^{(n)}\right)^{2} / d \mathfrak{A}_{0}\right)$ and $\int\left(\left(d \mathfrak{A}^{(n)}\right)^{2} / d \mathscr{A}_{0}\right)$ bounded in $n$, and $\lim _{n} \mathfrak{F}^{(n)}=\mathfrak{F}, \lim _{n} \mathfrak{F}^{(n)}=\mathbb{H}$. The bilinear form $A(\mathfrak{F}, \mathfrak{A})$ is completely continuous if

$$
\lim _{n \rightarrow \infty} A\left(\mathfrak{F}^{(n)}, \mathfrak{A}^{(n)}\right)=A(\mathfrak{F}, \mathfrak{F}) \text {. }
$$

If $A$ ( $\mathfrak{F},(\mathfrak{S})$ is completely continuous, the bilinear form $B(x, y)$ is completely continuous.

\section{Systems of linear equations. Completely continuous Case}

Let $A$ and $B$ be limited matrices connected by the relations (7) and (8), where $\left\{\mathfrak{P}_{i}\right\}$ is a closed orthogonal system with basis function $\mathfrak{A}_{0}$. If $\left\{m_{i}\right\}$ is a sequence of finite norm, the system of functions $\mathfrak{U}$ defined by

$$
\mathfrak{U}=\sum_{k} m_{k} \mathfrak{B}_{k}
$$

is integrable $H\left(\mathfrak{U}_{0}\right)$, and the sequence $\left\{m_{i}\right\}$ is expressed in terms of $\mathfrak{U}$ by

$$
m_{i}=\int \frac{d \mathfrak{B}_{i} d \mathfrak{U}}{d \mathfrak{U}_{0}} .
$$

The relations (9) and (10) establish a one-to-one correspondence between the solutions $\mathfrak{U}$, integrable $H\left(\mathfrak{A}_{0}\right)$, of the system of linear functional equations

$$
\mathfrak{U}=A[\mathfrak{U}] \text {, }
$$

* Hellinger and Toeplitz, Theorie der unendlichen Matrizen, $\$ 10, \mathrm{M}$ a t h e m a t i s c he Annalen, 1910, vol. 69. 
and the solutions $\left\{m_{i}\right\}$ of finite norm of the system of equations

$$
m_{i}=\sum_{k} b_{i k} m_{k} \quad(i=1,2, \cdots) .
$$

Multiply both sides of (12) by $\mathfrak{P}_{i}$, sum with respect to $i$, then in the right hand side substitute for $m_{i}$ from (10), and we obtain (11). Similarly we may pass from (11) to (12).

There is a one-to-one correspondence between the solutions of the adjoint systems

$$
\mathfrak{W}=A^{\prime}[\mathfrak{W}]
$$

$$
n_{i}=\sum_{k} b_{k i} n_{k}
$$

also between the solutions of the corresponding non-homogeneous equations

$$
\overline{\mathfrak{u}}=\mathfrak{F}+A[\overline{\mathfrak{u}}]
$$

where $\mathfrak{F}$ is integrable $H\left(\mathfrak{A}_{0}\right)$, and

$$
\bar{m}_{i}=c_{i}+\sum_{k} b_{i k} \bar{m}_{k},
$$

where $\left\{c_{\imath}\right\}$ is of finite norm, and $\mathfrak{F}=\Sigma_{k} c_{k} \mathfrak{B}_{k}$.

From the known theorems* concerning the solutions of (12), (14), and (16), we obtain the following theorem, if we understand by a solution, one which is integrable $H\left(\mathfrak{A}_{0}\right)$.

Theorem 2. Let the matrix $A=\left(a^{(a, \beta)}\left(E, E^{\prime}\right)\right)$ be limited with respect to $\mathfrak{A}_{0}$, and the bilinear form $A$ ( $\mathfrak{F},(\mathfrak{S})$ be completely continuous. If the homogeneous equations (11) have no non-trivial solutions, the non-homogeneous equations (15) have one and only one solution for every $\mathfrak{F}$ integrable $H\left(\mathfrak{A}_{0}\right)$. The homogeneous equations (11) can have only a finite number of linearly independent non-trivial solutions. If the equation (11) has $n$ linearly independent non-trivial solutions $\mathfrak{U}_{i}(E)$, the adjoint equation (13) has $n$ linearly independent non-trivial solutions $\mathfrak{W}_{\mathfrak{3}}(E)$, and the non-homogeneous equation (15) has solutions when and only when

$$
\int \frac{d \mathfrak{F} d \mathfrak{W}_{i}}{d \mathfrak{H}_{0}}=0 \quad(i=1,2, \cdots, n) .
$$

When $I_{a}$ consist of points in a space of one dimension, $a_{0}^{(1)}$ is formed from a monotonic non-decreasing function, constant except for a finite or denumerably infinite set of points of discontinuity in $I$, and $a_{0}^{(a)}(\alpha>1)$ formed

* Hilbert, D., Grundzüge einer allgemeinen Theorie der linearen Integralgleichungen, pp. 164-170. 
from continuous, monotonic non-decreasing functions, ${ }^{*}$ the equation (11) takes the form

$$
\begin{gathered}
x_{i}=\sum_{k} a_{i k} x_{k}+\sum_{\beta=2}^{\infty} \int_{a}^{b} \frac{d a_{i}^{(1, \beta)}(t) d u^{(\beta)}(t)}{d a_{0}^{(\beta)}(t)} \\
\Delta u^{(a)}(s)=\sum_{k} \Delta a_{k}^{(a, 1)}(s) x_{k}+\sum_{\beta=2}^{\infty} \int_{a}^{b} \frac{d_{t} \Delta a^{(\alpha, \beta)}(s, t) d u^{(\beta)}(t)}{d a_{0}^{(\beta)}(t)},
\end{gathered}
$$

in which the unknowns are $\left\{x_{i}\right\}$ and $\left\{u^{(a)}(s)\right\}$. As an illustration of (17) in which the form $A(\mathfrak{F},(\mathfrak{S )})$ is completely continuous, we give the following. The range of $\alpha$ and $\beta$ is that of a single element; of $i$ and $k$, a finite number $n$ of elements; $a_{i k}=1$ for $i=k, a_{i k}=0$ for $i \neq k ; a_{i}^{(1,2)}(s)$ are continuous functions and have continuous first derivatives $K_{i}(s) ; a_{i}^{(2,1)}(s)$ are continuous functions and have continuous first derivatives $L_{i}(s) ; a^{(2,2)}(s, t)$ is continuous in $s$ and $t$, and has continuous first and second partial derivatives, $\partial a^{(2,2)} / \partial t$ and $\left(\partial^{2} a^{(2,2)} / \partial s \partial t\right)=K(s, t) ; a_{0}^{(2)}(s)=s$. The equations (17) are in this case equivalent to

$$
\begin{aligned}
0 & =\int_{a}^{b} K_{i}(t) \phi(t) d t \quad(i=1,2, \ldots, n) . \\
\phi(s) & =\sum_{k=1}^{n} L_{k}(s) x_{k}+\int_{a}^{b} K(s, t) \phi(t) d t,
\end{aligned}
$$

where $\left\{x_{i}\right\}$ and $\phi(s)$ are the unknowns. The adjoint system is

$$
\begin{aligned}
0 & =\int_{a}^{b} L_{i}(t) \psi(t) d t, \\
\psi(s) & =\sum_{k=1}^{n} K_{k}(s) y_{k}+\int_{a}^{b} K(t, s) \psi(t) d t,
\end{aligned}
$$

where $\left\{y_{i}\right\}$ and $\psi(s)$ are the unknowns. The system of non-homogeneous equations corresponding to (18) has the form

$$
\begin{aligned}
0 & =c_{i}+\int_{a}^{b} K_{i}(t) \bar{\phi}(t) d t \quad(i=1,2, \cdots, n), \\
\bar{\phi}(s) & =f(s)+\sum_{k=1}^{n} L_{k}(s) \bar{x}_{k}+\int_{a}^{b} K(s, t) \bar{\phi}(t) d t,
\end{aligned}
$$

and the necessary and sufficient conditions that it have solutions when (19) has solutions $y_{k i}, \psi_{k}(s)$, are

$$
\sum_{i=1}^{n} c_{i} y_{k i}+\int_{a}^{b} f(t) \psi_{k}(t) d t=0 \quad(k=1,2, \cdots, m) .
$$

* Radon, J., pp. 1321-1322, and Hildebrandt, T. H., On integrals related to and extensions of the Lebesgue integrals, p. 197, Bulletin of the American Mathe matical S o c i è t y, January, 1918. 
5. SYMMETRIC SYSTEMS OF LINEAR EQUATIONS

A limited matrix $A$ is symmetric if $a^{(a, \beta)}\left(E, E^{\prime}\right)=a^{(\beta, a)}\left(E^{\prime}, E\right)$, and then the matrix $B$ defined by (7) is also symmetric. When $B$ is symmetric and limited, there exists $\dagger$ a spectrum $s$, lying within a finite interval of the $\lambda$-axis which consists of real characteristic numbers $\lambda_{i}^{*}$, or of a continuous spectrum $\left(a^{*}, b^{*}\right)$ or of both, for the equations

$$
\begin{aligned}
\lambda^{*} l_{k}^{*} & =\sum_{j} b_{k j} l_{j}^{*}, \\
\int_{\Delta} \lambda d p_{k}^{*} & =\sum_{j} b_{k j} \Delta p_{j}^{*}(\lambda),
\end{aligned}
$$

where $\Delta$ is any subinterval of $\left(a^{*}, b^{*}\right)$. Let $\left\{L_{i}^{*}(x)\right\}$ be the characteristic linear forms corresponding to $\lambda_{i}^{*}$ and $\left\{d P^{*(a)}(\lambda ; x)\right\}$ the characteristic linear differential forms; they form an orthogonal system with basis functions $\left\{p_{0}^{* a}\right\}$, that is

$$
\begin{gathered}
\left(L_{i}^{*}, L_{k}^{*}\right)=\left\{\begin{array}{ll}
1 & i=k \\
0 & i \neq k
\end{array},\right. \\
\left(\Delta_{1} P^{*(a)}, \Delta_{2} P^{*(\beta)}\right)=\left\{\begin{array}{cc}
\Delta_{12} p_{0}^{*(a)} & \alpha=\beta \\
0 & \alpha \neq \beta
\end{array},\right. \\
\left(L_{i}^{*}, \Delta P^{*(a)}\right)=0,
\end{gathered}
$$

where $\Delta_{1}$ and $\Delta_{2}$ are any two subintervals of $\left(a^{*}, b^{*}\right)$, and $\Delta_{12}$ is the subinterval common to them. If for some values of $\alpha$ we allow $r_{i}^{(a)}(\lambda)$ to be discontinuous functions, constant except at $\lambda_{i}^{*}$ where they have finite discontinuities and the corresponding basis functions $r_{0}^{(a)}(\lambda)$ have unit discontinuities, the two equations (20) may be expressed by the one equation

$$
\int_{\Delta} \lambda d r_{i}^{(a)}(\lambda)=\sum_{j} b_{k j} \Delta r_{j}^{(a)}(\lambda),
$$

where $\Delta$ is any subinterval of the spectrum $s$; and the orthogonal relations (21) by

The relation

$$
\left(\Delta_{1} R^{(a)}, \Delta_{2} R^{(\beta)}\right)=\left\{\begin{array}{cc}
\Delta_{12} r_{0}^{(a)} & \alpha=\beta \\
0 & \alpha \neq \beta
\end{array}\right.
$$

$$
l^{(a, \beta)}(\lambda, E)=\sum_{i} r_{i}^{(\alpha)}(\lambda) p_{i}^{(\beta)}(E)
$$

establishes a one-to-one correspondence between the solutions of (22), and the solutions $l^{(a, \beta)}(\lambda, E)$, integrable $H\left(\mathfrak{A}_{0}\right)$ for every $\alpha$ and $\lambda$, of

$$
\int_{\Delta} \lambda d_{\lambda} \mathfrak{R}_{\lambda}^{(a)}=A\left[\Delta \mathfrak{R}_{\lambda}^{(a)}\right]
$$

where $\Delta$ is any subinterval of $s$. For the characteristic numbers $\lambda_{i}^{*}$, the equation (24) becomes

$$
\lambda_{i} \mathfrak{R}_{i}=A\left[\mathfrak{R}_{i}\right] .
$$

† Hilbert, D., l. c., pp. 109-174; and Hellinger, E., l. c., pp. 210-271. 
The orthogonal properties (23) yield the following orthogonal properties for $\mathfrak{R}_{\lambda}^{a}$

$$
\int \frac{d \Delta_{1} \Re_{\lambda}^{a} d \Delta_{2} \Re_{\lambda}^{\beta}}{d \Re_{0}}=\left\{\begin{array}{cc}
\Delta_{12} r_{0}^{(a)}(\lambda) & \alpha=\beta \\
0 & \alpha \neq \beta
\end{array}\right.
$$

From the expansion of $B$ in terms of the characteristic forms, we obtain the expression of $A$ in terms of $\mathfrak{R}_{\lambda}^{a}$

$$
a^{(a, \beta)}\left(E, E^{\prime}\right)=\int_{s} \lambda \frac{d_{\lambda}{R_{E}^{\prime a}}_{d_{\lambda} R_{E^{\prime}}^{\prime \beta}}}{d \Re_{0}}
$$

The results may be summarized in the following theorem.

Theorem 3. If the matrix $A=\left(a^{(a, \beta)}\left(E, E^{\prime}\right)\right)$ is symmetric, limited with respect to $\mathfrak{A}_{0}$, and not identically zero, there exists a spectrum, lying within a finite interval of the $\lambda$-axis, which consists of characteristic numbers, or of a continuous spectrum, or of both, and for which the equations (24) have solutions, integrable $H\left(\mathfrak{A}_{0}\right)$ for every $\alpha$ and $\lambda$. The solutions form an orthogonal system (25), and the matrix $A$ may be expressed in terms of them in the form (26).

By applying results obtained by us* for linear equations with an unsymmetric matrix of coefficients, we obtain the following theorem.

Theorem 4. If the limited matrix $A=\left(a^{(\alpha, \beta)}\left(E, E^{\prime}\right)\right)$ is not identically zero, and there exists a limited symmetric matrix $T=\left(t^{(a, \beta)}\left(E, E^{\prime}\right)\right)$ such that

$$
T(\mathfrak{F}, \mathfrak{F}) \geqq 0
$$

the equality sign holding only for $\mathfrak{F} \equiv 0$, and such that the product matrix

$$
C=A T
$$

is symmetric, then there exists associated with $A$ a spectrum, lying within a finite interval of the $\lambda$-axis, which consists of characteristic numbers, or of a continuous spectrum, or of both, and for which the equation

has solutions.

$$
\int_{\Delta} \lambda d \mathfrak{R}_{\lambda}^{a}=A\left[\Delta \mathfrak{R}_{\lambda}^{a}\right]
$$

Let $s_{i k}=T\left(\mathfrak{B}_{i}, \mathfrak{B}_{k}\right)$, then $B S$ is a symmetric limited matrix, and the necessary conditions on $B$ are fulfilled.

\section{General orthogonal matrices}

In this section we define a general orthogonal matrix $L=\left(l^{(a, \beta)}\left(E, E^{\prime}\right)\right)$, and derive some properties. The orthogonal matrices represented by the relations (1), (4), (21), and (25) are special cases.

Let the functions $l^{(\alpha, \beta)}\left(E, E^{\prime}\right)$ be absolutely additive in $E$ and $E^{\prime} ; a_{0}^{(\alpha)}(E)$ and $b_{0}^{(\beta)}\left(E^{\prime}\right)$ absolutely additive and monotonic; $\mathfrak{R}_{E}^{a}$ integrable $H\left(\mathfrak{B}_{0}\right)$ for

${ }^{*}$ Pell, A. J., Linear equations with unsymmetric systems of coefficients, these $\mathrm{T} \mathbf{r}$ a $\mathrm{n}$ s a ct i o $\mathrm{n}$ s, January, 1919. 
every $\alpha$ and $E$. The matrix $L$ forms an orthogonal matrix with respect to $\mathfrak{A}_{0}$, if (27)

From

$$
L L^{\prime}=\Delta\left(\mathfrak{A}_{0}\right) \text {. }
$$

$$
\int \frac{\left(d\left\{\mathfrak{F}-\sum_{a=1}^{m} \sum_{i=1}^{n} \frac{\mathfrak{R}_{E_{i}}^{a} L_{E_{i}}^{a}[\mathfrak{F}]}{a_{0}^{(a)}\left(E_{i}\right)}\right\}\right)^{2}}{d \mathfrak{B}_{0}} \geqq 0,
$$

it follows that for every $\mathfrak{F}$ integrable $H\left(\mathfrak{B}_{0}\right)$

$$
\sum_{a=1}^{m} \sum_{i=1}^{n} \frac{\left(L_{E_{i}}^{a}[\mathfrak{F}]\right)^{2}}{a_{0}^{(\alpha)}\left(E_{i}\right)} \leqq \int \frac{(d \mathfrak{F})^{2}}{d \mathfrak{B}_{0}}
$$

From this, Bessel's inequality is obtained,

$$
\int \frac{(d L[\mathfrak{F}])^{2}}{d \mathfrak{A}_{0}} \leqq \int \frac{(d \mathfrak{F})^{2}}{d \mathfrak{B}_{0}}
$$

which expresses that $L[\mathfrak{F}]$ is integrable $H\left(\mathfrak{A}_{0}\right)$. As a special case we have that $\mathfrak{R}_{E}^{\prime a}$ is integrable $H\left(\mathfrak{A}_{0}\right)$ for every $\alpha$ and $E$, therefore

$$
L^{\prime}[L[\mathfrak{F}]]
$$

exists for every $\mathfrak{F}$ integrable $H\left(\mathfrak{B}_{0}\right)$.

The system $\mathfrak{R}_{E}^{a}$ is closed if there exists no system $\mathbb{S}$, not identically zero, integrable $H\left(\mathfrak{B}_{0}\right)$, and such that

for every $\alpha$ and $E$.

$$
L_{E}^{a}[(\$)]=0
$$

If the matrix $L$ is orthogonal and $\mathfrak{R}_{E}^{a}$ is closed, then for every $\mathfrak{F}$ integrable $H\left(\mathfrak{B}_{0}\right)$

and also

$$
L^{\prime}[L[\mathfrak{F}]]=\mathfrak{F},
$$

$$
L^{\prime} L=\Delta\left(\mathfrak{B}_{0}\right) \text {. }
$$

If the matrix $L$ is composed of $l_{i k}, p_{i}^{(a)}(s), \pi_{k}^{(\beta)}(t)$, and $\Lambda^{(a, \beta)}(s, t)$, the orthogonal condition (27) becomes

$$
\begin{gathered}
\sum_{j} l_{i j} l_{k j}+\sum_{\beta} \int_{a}^{b} \frac{d \pi_{i}^{(\beta)} d \pi_{k}^{(\beta)}}{d b_{0}^{(\beta)}}= \begin{cases}1 & i=k \\
0 & i \neq k\end{cases} \\
\sum_{j} \Delta_{1} p_{j}^{\left(\alpha_{1}\right)}(s) \Delta_{2} p_{j}^{\left(\alpha_{2}\right)}(s)+\sum_{\beta} \int_{a}^{b} \frac{d t \Delta_{1} \Lambda^{\left(\alpha_{1}, \beta\right)}(s, t) d_{t} \Delta_{2} \Lambda^{\left(\alpha_{2}, \beta\right)}(s, t)}{d b_{0}^{(\beta)}(t)} \\
=\left\{\begin{array}{cc}
\Delta_{12} a_{0}^{\left(\alpha_{1}\right)} & \alpha_{1}=\alpha_{2} \\
0 & \alpha_{1} \neq \alpha_{2}
\end{array}\right. \\
\sum_{j} l_{i j} \Delta p_{j}^{(\beta)}(s)+\sum_{\beta} \int_{a}^{b} \frac{d \Lambda^{(\alpha, \beta)}(s, t) d \pi_{i}^{(\beta)}(t)}{d b_{0}^{(\beta)}(t)}=0,
\end{gathered}
$$

and (28) has a similar form.

Bryn Mawr College 